Relations industrielles

Industrial Relations

\title{
La participation des travailleurs aux bénéfices est-elle un cadeau?
}

\section{Gérard Dion}

Volume 3, numéro 9, mai 1948

URI : https://id.erudit.org/iderudit/1023620ar

DOI : https://doi.org/10.7202/1023620ar

Aller au sommaire du numéro

Éditeur(s)

Département des relations industrielles de l’Université Laval

ISSN

0034-379X (imprimé)

1703-8138 (numérique)

Découvrir la revue

Citer cet article

Dion, G. (1948). La participation des travailleurs aux bénéfices est-elle un cadeau ? Relations industrielles / Industrial Relations, 3(9), 132-136.

https://doi.org/10.7202/1023620ar

Tous droits réservés (C Département des relations industrielles de l’Université Laval, 1948
Ce document est protégé par la loi sur le droit d'auteur. L'utilisation des services d'Érudit (y compris la reproduction) est assujettie à sa politique d'utilisation que vous pouvez consulter en ligne.

https://apropos.erudit.org/fr/usagers/politique-dutilisation/ 
entreprise pour y parvenir. Le chiffre d'affaires augmentant, il suffisait de lier à lui le taux des salaires pour que ceux-ci augmentent automatiquement. Au moment de l'établissement du système dans l'entreprise, il était même possible de redresser partiellement le retard des salaires en établis-

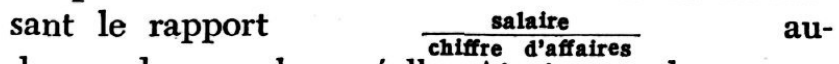
dessus de sa valeur réelle. Ainsi, sous le couvert d'une formule qui n'était nullement suspect, il était possible de tourner les ordonnances en vigueur, ce qui rendit les plus réels services. De même, les quelques augmentations de prix qui eurent lieu se répercutaient presque invisiblement sur les salaires. Il n'est pas question bien entendu d'expliquer des augmentations de $50 \%$ et même de $100 \%$ par ce seul coup de pouce initial. Il convenait, toutefois, de le mentionner.

D'autre part, le dessein profond du salaire proportionnel est de distribuer la part du travail, c'est-à-dire dans l'esprit de M. Schueller, ce qui revient au salarié d'une augmentation du chiffre principalement causée par le machinisme. (Nous ne croyons pas trahir sa pensée en disant "principalement», puisque son but avoué est de parer aux crises de surproduction, indiscutablement d'autant plus graves que le machinisme est plus développé).

Or, dans la plupart des applications faites, ce n'est pas un effort de mécanisation que traduisent les augmentations de salaire, mais presque uniquement un effort humain, d'ingéniosité, de temps gagné, de bonne volonté.

On peut donc affirmer que, de toute façon, il résulte du salaire proportionnel un accroissement de rendement et des économies de temps qui profitent à tous. Toutefois si la formule a cette portée économique, à cause de ce caractère même on peut donter si elle a une portée sociale aussi considérable que la simple participation aux bénéfices.

\section{LA PARTICIPATION DES TRAVAILLEURS AUX BÉNÉFICES EST - ELLE UN CADEAU? \\ Gérard DION}

Beaucoup de bonnes gens se sont émues à la suite de déclarations apparemment contradictoires faites par deux propagandistes de groupements d'inspiration catholique. Dans une réunion à Chicoutimi devant des industriels on a catégoriquement affirmé : «Les bénéfices d'une entreprise appartiennent de droit au patron et servent ordinairement pour la plus grande partie, à son expansion et à la création de nouveaux emplois pour les travailleurs. On ne connaît pas aux employés de droit strict au bénéfice. Si un patron veut librement partager ses bénéfices, il peut le faire. Est recommandée alors une prudente formule d'intéressement. Quant à ce qu'on appelle la co-gestion on recommande la formule des comités de coopération avec voix consultative, mais on est formellement opposé à la co-gestion stricte avec voix délibérative, la direction de l'entreprise appartenant de droit au patron. » ${ }^{1}$ Dans la même région, à Kénogami, un organisateur ouvrier déclarait au cours d'une journée syndicale : «Le grand défaut de la société actuelle, c'est que tout est ordonné non au bien public, mais au bénéfice de quelques-

(1) L'Action Catholique, 29 janvier 1948. uns. L'ouvrier a droit non seulement à un salaire vital, mais aussi à la participation aux bénéfices... le correctif qu'il faut apporter à la construction de la société nouvelle, c'est d'accorder aux ouvriers la participation aux bénéfices, la co-gestion et la co-propriété des usines où ils travaillent. C'est le moyen et pratiquement le seul pour éviter le com-

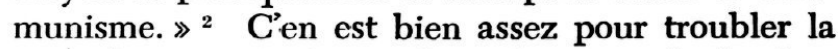
paix des esprits qui veulent s'inspirer de la doctrine chrétienne. Lequel des deux a raison, se demande-t-on avec bon sens? Lequel des deux est dans la ligne de la pensée pontificale?

Dans la chaleur de la discussion, au cours d'une envolée oratoire, surtout quand on tâche de ne pas déplaire à son auditoire et de l'entraîner à sa suite, on ne peut exiger des propagandistes d'entrer dans les distinctions et les subtilités des théoriciens. C'est habituellement celui qui est le plus catégorique et qui affirme le plus fort qui apporte le morceau. Cette constatation de sens commun se réalise tout bonnement dans ce cas-ci. Nos deux orateurs défendent chacun leur point de

(2) Le Progrès du Saguenay, 11 mars 1948. 
vue, tous deux s'appuient sur des textes pontificaux; tous deux ont à la fois raison et tort.

La vénération que l'on doit accorder aux encycliques sociales ne dispense personne de scruter à fond la pensée de celui à qui $\mathrm{N}$. S. a dit : «Celui qui vous écoute, m'écoute; celui qui vous méprise, me méprise » et de chercher le pourquoi des déclarations du chef de l'Eglise. C'est le seul moyen d'interpréter légitimement les encycliques et de ne pas leur faire dire ce qu'elles ne disent pas.

Dans une encyclique, le Pape, s'adressant à l'Eglise universelle, parle en docteur et en pasteur. Le texte sur lequel se basent ces deux antagonistes en est une belle illustration.

Quand Pie XI dit : «Commençons par relever la profonde erreur de ceux qui déclarent essentiellement injuste le contrat de louage de travail et prétendent qu'il faut lui substituer un contrat de société; ce disant, ils font, en effet, gravement injure à Notre Prédécesseur, car l'Encyclique Rerum novarum non seulement admet la légitimité du salariat, mais s'attache longuement à le régler selon les normes de la justice » ${ }^{3}$, il parle alors en docteur, et avec toute son autorité, il rappelle une vérité conforme à la raison. Quand il ajoute : «Nous estimons cependant plus approprié aux conditions présentes de la vie sociale de tempérer quelque peu, dans la mesure du possible, le contrat de travail par des élèments empruntés au contrat de société. C'est ce que l'on a déjà commencé à faire sous des formes variées, non sans profit sensible pour les travailleurs, et pour les possesseurs du capital. Ainsi les ouvriers et employés ont été appelés à participer en quelque manière à la propriété de l'entreprise, à sa gestion ou aux profits quelle apporte,$^{4}$ il parle ici en pasteur soucieux du bielı des personnes confiées à sa charge. Dans le premier cas, c'est un absolu, qui reste toujours vrai, indépendamment des circonstances; dans le second, c'est un relatif qui tient compte des contingences particulières à l'époque où ce souhait a été exprimé et cette directive donnée.

Les ouvriers ont-ils un droit strict aux bénéfices? Cette question peut être considérée à deux moments différents. Avant la passation de tout contrat, c'est-à-dire en envisageant la nature mème du travail effectué avec d'autres et le droit des

(3) Pie XI, Quadragesimo Anno, no 71.

(4) id. travailleurs aux fruits de leur travail; après qu'un contrat est intervenu pour déterminer concrètement la répartition des fruits du travail.

Les socialistes soutiennent que le contrat de salariat est contre nature et que seul le contrat de société est admissible. Ils s'appuient sur l'inaliénabilité du travail et des fruits qui en découlent. Ils veulent que tout le fruit du travail, tous les profits retournent aux travailleurs parce que le capital de lui-même est improductif et seul le travail le rend fécond.

La doctrine catholique est tout autre. Parce que travail et capital concourent, - de façon diverse, il est vrai, - à produire un bien utile, tous deux ont des droits sur les profits que procure l'échange de ce bien. Le capital n'est donc pas seul à avoir des droits sur le profit. En effet, réduit à ses seules forces, le mieux qu'il puisse faire, c'est de se conserver. S'il produit un bien qui rapporte un bénéfice, c'est qu'il est uni au travail. Ce sont donc les deux éléments, capital et travail qui ont des droits sur les profits et qui doivent partager la plus-value. «Il serait donc radicalement faux de voir, soit dans le seul capital, soit dans le seul travail, la cause unique de tout ce que produit leur effort combiné; c'est bien injustement que l'une des parties contestant à l'autre toute efficacité, en revendiquerait pour soi tout le fruit. $»^{5}$

Les RR. PP. Desbuquois et Bigo précisent comme suit ces exigences : «En réalité, le capital n'est pas seul habilité à recevoir le profit. Non seulement, il doit laisser le travail $\epsilon$ n recevoir sa part (s'il y a vraiment profit); mais il doit accepter de ne rien recevoir du tout, s'il ne reste rien après le paiement du salaire minimum et le service de l'amortissement et des réserves. Le droit du capital au profit demeure aléatoire. Par l'essence même de son rôle, le capital ne peut, en effet, revendiquer autre chose que de participer au partage d'un profit hypothétique. On ne saurait trop insister sur cette idée méconnue. Tandis que la rémunération du travail comporte le paiement d'un minimum indépendant des aléas de l'affaire et qui ne se calcule pas en fonction d'un profit à partager, la rémunération du capital, qu'elle lui soit payée sous forme de dividendes ou d'intérêts, est au contraire essentiellement un gain conditionné. Le caractère de son gain est, en effet, commandé par le caractère de son rôle : agent qui ne peut contribuer à la production que dans une association avec le travail, association qui comporte, naturellement, un risque. 
Ce sort inégal du travail et du capital dans l'entreprise heurterait moins, si l'on adoptait ici la vision résolument «humaniste ${ }^{6}$ qui est celle de l'Eglise. Deux réalités sont en présence : l'une, le capital, est une chose, du travail cristallisé peutêtre, mais à présent, une simple chose matérielle, morte; l'autre, le travail, est un être vivant, l'acte d une personne humaine qui a droit à la vie. Or, les choses sont faites pour les personnes; leur fonction est d'être au service de la vie. Service tantôt rémunéré, si l'entreprise réussit; service parfois stérile, parfois mortel, en cas d'insuccès ou de ruine. Le capital n'a donc pas pour destinée de gagner en servant, c'est-à-dire de ne servir qu'à la condition de gagner; mais sa fin ultime est toujours de servir, fût-ce, dans certaines circonstances, au prix même de son existence. Par analogie, il en est du capital engagé dans l'entreprise comme de la propriété privée : celle-ci cesse d'exister, elle s'évanouit et disparaît entre les mains de son possesseur dans le cas d'extrême nécessité du prochain, c'està-dire devant une exigence absolue de la vie en danger de mort. Ainsi le capital ira-t-il éventuellement jusqu'au sacrifice plus ou moins complet de lui-même au profit de la vie humaine.

Si l'on va à la source de la prétention du capital à l'exclusivité de la plus-value, on découvre ainsi l'assimilation abusive entre le capital et le travail, la mise sur le même plan d'une chose et d'une personne humaine. Le capital aurait, au même titre que la personne, le droit de se conserver, de s'agrandir, tandis qu'en vérité, ce droit n'est que relatif, subordonné aux exigences de la personne. Cette erreur initiale atteint sa suprême gravité dans le mammonisme absolu qui confère au capital le droit de croître sans aucun égard à la vie du travailleur. Il n'est, grâce à Dieu, point de catholiques qui adoptent cette conception monstrueuse. Mais la cure de désintoxication est lente, et malgré l'enseignement pontifical, le venin mammoniste, dont s'est nourrie l'économie moderne, a laissé des traces dans bien des esprits.

La conclusion s'impose: le droit au profit n'est pas un attribut exclusif du capital. Dès lors, il apparaît qu'il est attaché au titre de membre de la communauté d'entreprise, titre qui s'acquiert aussi bien par le travail que par l'apport d'argent ${ }^{\text {? }}$. Il doit donc y avoir, en principe, dans le salaire payé au travail, une somme qui corresponde à son

(6) "Humaniste" doit s'entendre ici dans le sens d'humain puisqu'il ne s'agit pas d'une fin absolue, mais relative. - G.D.

(7) Cette argumentation ne s'appuie nullement, on le voit, sur l'idée discutée, et très discutable, d'un droit de propriété du salarié sur les objets fabriqués dans l'usine. (Note des auteurs) droit sur le profit et qui vienne en supplément d'un minimum vital, familial, humain, inconditionné.» ${ }^{8}$ La justice n'exige pas nécessairement que ce droit des travailleurs et du capital sur les profits de l'entreprise soit déterminé concrètement par un contrat de société, comme le prétendent les socialistes. Elle n'exige pas davantage qu'il le soit par un contrat de salariat. Mais, dans tout contrat de travail, on doit tenir compte de ces droits des travailleurs sur le profit. Si l'on analyse et dissèque le contrat de salariat, on verra comment il peut être licite. En somme, ce contrat en contient deux implicitement: un par lequel le travailleur loue son activité pour une somme fixe, soit, par exemple un dollar l'heure, l'autre par lequel il renonce en faveur de l'employeur son droit sur les profits moyennant une summe fixe indépendante des aléas, soit, par exemple, vingt cents l'heure. Le contrat de salariat comporterait donc la somme des deux, soit un dollar vingt l'heure, l'employeur gardant tous les bénéfices, s'il en reste; de son côté, le travailleur est assuré de recevoir sa rémunération forfaitaire. Aucun doute sur la licéité de ce contrat. Mais il y a une marge entre affirmer que le contrat de salariat est licite et affirmer qu'il soit la seule forme de contrat de travail licite. Les tenants aveugles du contrat de salariat oublient parfois que le contrat de salariat est un contrat, i.e. qu'il suppose essentiellement l'accord des parties, et qu'il n'est qu'une des formes de contrat de travail. Les moralistes savent que le contrat de travail se divise en contrat d'entreprise, contrat de salariat, contrat de société, etc. Ce n'est pas parce que nous vivons dans un régime économique où prédomine le contrat de salariat que l'on est en droit de lui assurer le monopole. D'ailleurs, les faits sont là qui nous démontrent que, même dans le régime économique actuel, les autres formes de contrat de travail sont en vigueur ici et là. Le contrat de société et le contrat de salariat, dans leur détermination concrète, donnent naissance au droit strict des employeurs et des travailleurs. Ce n'est donc pas une er:eur d'affirmer que les travailleurs ont un droit strict aux profits. C'est une assertion incomplète, imprécise. Le salaire forfaitaire, la rémunération minimum avec participation aux profits sont tous

(8) G. Desbuquois, P. Bigo. Les Reformes de l'Entreprise et la pensée chrétienne, Spes, p. 11. On consultera aussi avec intérêt:

R. Kothen, Problèmes sociaux actuels, Bibliotheca Mechlinieusis, Desclée, de Brouwer, 1946, pp. 63-66.

R.P. Desquerat, s.j., Propriété et entreprise, dans Semaine sociale de France, Toulouse, 1945, pp. 101.

R.P. Chenu, o.p. La notion de profit et les principes chrétiens, idem, pp. 125-139. 
deux des droits stricts. Tout dépend de la soriè de contrat sur lequel se sont entendus employeurs et travailleurs.

Si certains employeurs ne veulent pas négocier de contrat de société et réusissent à se procurer de la main-d'oeuvre qui consente à accepter le contrat de salariat, celle-ci, n'aura plus, une fois cette entente faite, un droit strict de réclamer une participation aux profits. Et par contre, si les travailleurs, qui jusqu'ici étaient rémunérés à forfait, décident de ne plus louer leurs services à cette condition et exigent un contrat de société, unc fois cette nouvelle forme de contrat de travail acceptée, il auront un droit strict aux profits. C'est ce qui faisait dire au Cardinal Suhard: "Les ouvriers ont pu jusqu'ici, par contrat de salaire pur et siniple, se dessaisir d'une partie de leurs droits en échiange d'une assurance tacite contre les risques. ils veulent aujourd'hui en France, retrouver ces droits pour sortir de leur état de dépendance: ictir demande est normale. Le contrat de salaire pur et simple était juste, mais il était libre: nul rn'a le droit de l'imposer à ceux qui n'en veulent plus. Qu'on cherche donc des solutions nouvelles, avec une généreuse et prudente hardiesse.» ${ }^{9}$

Reste maintenant à étudier s'il est préférable pour le bien de tous de choisir le contrat de salariat ou le contrat de société ou des contrats mixtes. Ce n'est plus une question de vrai ou de faux, de juste ou d'injuste, mais simplement une question de prudence qui se résout en tenant compte des circonstances particulières à une époque, à un pays, à l'évolution de la civilisation, etc. Cet aspect est bien différent du premier. Quant le Pape se prononce ici, ce n'est plus en tant que docteur, mais en tant que pasteur qui peut, soit donner des ordres ou des conseils, ou simplement exprimer ses préférences. Il n'y a aucun doute que les fidèles qui révèrent en lui le chef éclairé, sage, indépendant de tout intérêt particulier doivent se faire un devoir d'en tenir compte. N'oublions cependant pas que dans ce cas, les circonstances jouent un ròle considérable. Les Souverains Pontifes, non seulement considèrent qu'elles peuvent changer, mais ils ont pris la peine de modifier, de préciser, d'adapter leurs directives; ils ont même louangé les évêques, les théologiens qui se sont efforcés de le faire dans leur milieu respectif. ${ }^{10}$

(9) L'Enseignement de l'Eglise sur la propriété. Lettre pastorale de S.E. le Cardinal Suhard, archevêque de Paris, Editions jocistes, Bruxelles, pp. 39-40

(10) "Par leurs paroles, par leurs écrits, et Léon XIII et ses successeurs ont continué à prêcher avec insis-
En 1931, le pape Pie XI au sujet du contrat de travail disait alors: « Nous estimons plus approprié aux conditions de la vie sociale de tempérer quelque peu, dans la mesure du possible, le contrat de travail par des éléments empruntés au contrat de société... » comme nous l'avons cité au début de cet article.

Admirons ici le sens pratique et la prudence pontificale. Pie XI ne donne pas un ordre, il constate un état de fait, il exprime un désir en louangeant ceux qui ont déjà fait des efforts pour le réaliser et d'avance il se réjouit de voir que l'on s'oriente dans ce sens. Il n'y a aucun doute que les catholiques qui, au lieu de marcher dans cette direction, font tout leur possible pour bloquer ces réformes et rebrousser un courant irrésistible, ne sont pas dans l'esprit de la pensée pontificale.

Mais ce texte remonte à dix-sept ans. Depuis ce temps-là, au point de vue économique et social, on a évolué. D'autres documents pontificaux ont été publiés, d'autres explicitations de la doctrine de l'Eglise ont été faites. La dernière guerre spécialement a fait avancer les choses considérablement. Le Pape Pie XII a cependant pris la peine de réaffirmer le souhait de son prédécesseur. En septembre 1944, il disait: «Là où la grande exploitation continue de se montrer plus heureusement productive, elle doit offrir la possibilité de tempérer le contrat de travail par un contrat de société ». (Cf. Encycl. Quadragesimo Anno) ${ }^{11}$

tance la doctrine sociale et économique de l'Encyclique Rerum novarum; ils n'ont pas cessé d'en presser l'application et l'adaptation aux temps et aux circonstances, faisant toujours preuve d'une sollicitude particulière et toute paternelle envers les pauvres et les faibles dont, en fermes pasteurs, ils se sont faits les défenseurs. Avec autant de science et de zèle, de nombreux Evêques ont interprété la même doctrine, l'ont éclairée de leurs commentaires et adaptée aux situations des divers pays, suivant les décisions et la pensée du Saint-Siège. Aussi n'est-il pas étonnant que, sous la direction du magistère ecclésiastique, des hommes de science, prêtres et laïques, se soient attachés avec ardeur à développer, selon les besoins du temps, les disciplines économiques et sociales, se proposant avant tout d'appliquer à des besoins nouveaux les principes immuables de la doctrine de l'Eglise. Ainsi s'est constituée, sous les auspices et dans la lumière de l'Encyclique de Léon XIII une science sociale catholique, qui grandit et s'enrichit chaque jour, grâce à l'incessant labeur des hommes d'élite que nous avons appelés les auxiliaires de l'Eglise. Et cette science ne s'enferme pas dans d'obscurs travaux d'école; elle se produit au grand jour et affronte la lutte, comme le prouvent excellemment l'enseignement, si utile et si apprécié, institué dans les universités catholiques, les académies et les séminaires, les congrès ou "semaines sociales", tenus tant de fois avec de si beaux résultats, les cercles d'études, les excellentes publications de tout genre si opportunément répandues." Pie XI, Quadragesimo Anno, 19-20-21.

(11) Pie XII, Message du ler septembre 1944. Cité par C. Bied-Charreton, La propriété privée dans les derniers messages pontificaux. Action Populaire, Spes, Paris, p. 15. 
Un commentateur officieux de sa pensée, après la Semaine Sociale de Strasbourg, le R. P. de Marco, dans la Civitta Catolica, écrivait un article spécialement pour remettre en place certains économistes catholiques qui favorisaient trop facilement la nationalisation et affirmait, dans son texte: «Une habile politique concernant les douanes et les impôts, une participation limitée au capital social, la discipline des financements, une législation sociale hardie qui comporte aussi, dans les limites imposées par le fonctionnement de l'entreprise, une large participation des travailleurs aux profits et da la gestion de la production, constituent un ensemble de mesures qui, bien appliquées, corrigent les abus du capitalisme privé sans tomber dans le capitalisme d'Etat plus funeste encore. ${ }^{12}$

En France, l'assemblée des cardinaux, archevêques et évêques faisait la déclaration suivante (1945): «Nous demandons ... la participation progressive des ouvriers à l'organisation du travail, de l'entreprise, de la profession et de la cité. L'ouvrier veut se sentir vraiment chez lui dans l'entreprise, pour un travail plus humain, où il aura pu engager sa responsabilité d'homme et mettre de bon gré avec courage, attention et conscience, toute son activité: il aspire à participer à la vie sociale et économique de l'entreprise ainsi qu'aux fruits de la production ... Pour réaliser ces progrès sociaux, des réformes de structure sont nécessaires: nous sommes prêts à les accueillir. Mais pour qu'elles soient plus efficaces, il importe que l'unité de commandement et que l'autorité du chef de l'entreprise, fondée sur la compétence et la moralité, soient pleinement reconnues, qu'il né soit pas porté atteinte au droits légitimement acquis, qu'il soit tenu compte avec prudence des capacités de l'économie nationale et que tout esprit de violence soit écarté.

«Sur le choix des systèmes techniques (participation aux bénéfices ou à la gestion, actions du travail, entreprises communautaires, etc.), l'Eglise n'a pas à se prononcer. Son rôle n'est pas d'organiser le temporel. Elle rappelle simplement, mais fortement, le principe d'une orientation de plus en plus nette vers le contrat de société. ${ }^{13}$

S.E. le Cardinal Suhard, dans un document que nous avons déjà cité, était très explicite.

(12) A DE MARCo, Nationalisation ou Corporation? Documentation Catholique, Tome 43, (1946) 1057-70.

(13) Cité par Romain, Une Révolution? Oui, mais laquelle! Turcoing, 1945, pp. 145-146.
D'autres évêques européens ne craignaient pas de souligner le besoin de réforme de structure. Chez nous, en 1941, les évêques de la Province de Québec rappelaient le texte de Quadragesimo Anno et dernièrement l'Evêque de Chicoutimi, dans une lettre sur le Sens Social, disait: «Les sociologues et les économistes constatent que le simple régime du salariat n'entretient pas assez chez l'ouvrier le souci du travail compétent et honnête, ne cultive généralement pas l'esprit d'initiative et le sens des responsabilités, n’assure pas une équitable distribution des profits de l'entreprise. Le Capital et le Travail trouveront grand avantage à faire évoluer leurs relations dans le sens de l'association, c'est-à-dire vers une participation plus effective des travailleurs à la vie de l'entreprise, à sa gestion et aux profits qui en résultent. »14

Nous croyons que, devant une telle unanimité de théologiens et de moralistes, qu'il est assez difficile de rester dans la ligne des enseignements de l'Eglise en prônant la conservation du pur contrat de salariat à une époque où il est déjà dépassé. Il vaut mieux travailler efficacement et prudemment à lui substituer graduellement, à mesure que les circonstances le permettent, un contrat de société. Autrement on ne pourra pas mériter les éloges que faisait Pie XI à ceux qui essaient de faire avancer les enseignements de l'Eglise. ${ }^{15}$

Affirmer donc que les travailleurs ont un droit strict aux bénéfices, c'est une assertion incomplète et facilement équivoque. Il faut bien situer le moment où l'on se place. Si c'est avant la passation d'un contrat de travail, c'est vrai; mais si les travailleurs ont déjà accepté de se départir de ce droit moyennant une compensation, ils ont uniquement un droit strict à cette compensation jusqu'à ce qu'ils changent les clauses de la convention.

Affirmer que les bénéfices appartiennent de droit strict à l'employeur seul et que s'il partage avec ses employés, c'est uniquement parce qu'il veut faire un beau geste, c'est ressusciter la vieille rengaine paternaliste qui oublie que le contrat de travail est une entente bilatérale et libre dans laquelle les travailleurs ont leur mot à dire autant que l'employeur.

(14) Lettre de S.E. Mgr Georges Melançon, le ler mars 1948.

(15) Q.A. 19-20-21, cité note 10. 